Internat. J. Math. \& Math. Sci.

Vol. 22, No. 1 (1999) 155-160

S 0161-1712<99>22155-2

(c) Electronic Publishing House

\title{
NUMERICAL SOLUTION OF INTEGRAL EQUATIONS WITH FINITE PART INTEGRALS
}

\author{
SAMIR A. ASHOUR
}

(Received 22 July 1994 and in revised form 29 March 1994)

\begin{abstract}
We obtain convergence rates for several algorithms that solve a class of Hadamard singular integral equations using the general theory of approximations for unbounded operators.
\end{abstract}

Keywords and phrases. Singular integral equations.

1991 Mathematics Subject Classification. 65D05, 45L05.

1. Introduction. In several physical problems in aerodynamics, hydrodynamics, and elasticity, one encounters integral equations of the form

$$
A x=\frac{1}{\pi} \int_{-1}^{1} \frac{\sqrt{1-\tau^{2}} x(\tau)}{(\tau-t)^{2}} d \tau+\frac{1}{\pi} \int_{-1}^{1} \sqrt{1-\tau^{2}} h(t, \tau) x(\tau) d \tau=y,
$$

where the first integral in (1.1) is a finite part integral [4]. Under suitable conditions on the kernel and the right-hand side, the convergence of Galerkin's method and several collocation methods, proposed by Ioakimidis [5] and Williams [9], has been discussed by Golberg [2, 3]. This author, also, used a classical Fredholm theory to establish the existence of a solution and likewise the basic tools necessary to discuss convergence. In this paper, we discuss the convergence of the mechanical quadratures method for solving (1.1) and the convergence of the least squares method for solving the Hadamard singular integral equation

$$
K x=\frac{1}{\pi} \int_{-1}^{1} \frac{\sqrt{1-\tau^{2}} x(\tau)}{(\tau-t)^{2}} d \tau+T(x, t)=y,
$$

where $T$ is the given continuous operator.

2. Least squares method. Let $X=L_{2, \rho}$ denote the space of square integrable functions with respect to $\rho=\sqrt{1-t^{2}}$. The inner product on $L_{2, \rho}$ is given by

$$
(\phi, \psi)_{\rho}=\frac{2}{\pi} \int_{-1}^{1} \rho(t) \phi(t) \psi(t) d t \quad \text { and } \quad\|\phi\|_{\rho}=\sqrt{(\phi, \phi)_{\rho}} .
$$

Let

$$
U_{m}(t)=\frac{\sin [(m+1) \arccos t]}{\sqrt{1-t^{2}}}, \quad m=0,1,2, \ldots
$$


denote the Chebyshev polynomials of the second kind. The solution $x$ is, now, approximated by

$$
x_{n}(t)=\sum_{k=1}^{n} \alpha_{k} U_{k-1}(t), \quad-1 \leq t \leq 1 .
$$

According to this method, we obtain a system of $n$ linear algebraic equations in $n$ unknowns

$$
\sum_{i=1}^{n} \alpha_{i}\left(K U_{i-1}, K U_{j-1}\right)_{\rho}=\left(y, K U_{j-1}\right)_{\rho}, \quad 1 \leq j \leq n .
$$

It is easy to prove that (2.4) is equivalent to

$$
\begin{aligned}
& \sum_{k=1}^{n} \alpha_{k}\left\{\left(T U_{k-1}, T U_{j-1}\right)_{\rho}-j\left(T U_{k-1}, U_{j-1}\right)_{\rho}-k\left(T U_{j-1}, U_{k-1}\right)_{\rho}\right\}+j^{2} \alpha_{j} \\
&=\left(y, K U_{j-1}\right)_{\rho}, \quad 1 \leq j \leq n .
\end{aligned}
$$

THEOREM 2.1. If the following conditions hold

(i) $y \in L_{2, \rho}, T$ is a continuous operator in $L_{2, \rho}$;

(ii) $\operatorname{ker} K=\{0\}$;

(iii) equation (1.2) has a solution $x^{*} \in L_{2, \rho}$ for a given $y \in L_{2, \rho}$, then for all $n \in \mathbb{N}$ equation (2.5) has a unique solution $\left\{\alpha_{k}^{*}\right\}_{1}^{n}$; and if

(iv) $\left\{K U_{i-1}\right\}$ is closed in $L_{2, \rho}$, then

$$
\left\|r_{n}\right\|_{\rho}=\left\|y-K x_{n}^{*}\right\|_{\rho} \longrightarrow 0 \quad \text { as } \quad n \rightarrow \infty, \quad x_{n}^{*}=\sum_{k=1}^{n} \alpha_{k}^{*} U_{k-1}(t)
$$

Proof. Since $\left\{U_{k-1}\right\}$ are linearly independent, then, from (ii), it follows that $\left\{K U_{k-1}\right\}$ are, also, linearly independent. Therefore, the system of equations (2.5) is non-singular and so, it has a unique solution for all $n$. Also, for $\beta_{k} \in \mathbb{R}$, we get

$$
\left\|r_{n}\right\|_{\rho}=\left\|y-K x_{n}^{*}\right\| \leq\left\|y-\sum_{i=1}^{n} \beta_{i} K U_{i-1}\right\|_{\rho} .
$$

If condition (iv) is satisfied, then $\left\|r_{n}\right\| \longrightarrow 0$ as $n \longrightarrow \infty$.

Now, we replace condition (ii) by the following condition:

(ii) $^{\prime} K: L_{2, \rho} \longrightarrow L_{2, \rho}$ has a left bounded inverse operator $K_{l}^{-1}$.

THEOrem 2.2. Assume that (i), (ii) ${ }^{\prime}$, (iii), and (iv) are satisfied, then $\left\|x_{n}^{*}-x^{*}\right\|_{\rho}=$ $O\left(\left\|r_{n}\right\|_{\rho}\right) \longrightarrow 0$ as $n \longrightarrow \infty$.

Proof. From (ii)' and (iii), we have $x^{*}-x_{n}^{*}=K_{l}^{-1} K\left(x^{*}-x_{n}^{*}\right)=K_{l}^{-1}\left(y-K x_{n}^{*}\right)$, then $\left\|x_{n}^{*}-x^{*}\right\|_{\rho} \longrightarrow 0$ as $n \longrightarrow \infty$.

3. Mechanical quadratures methods. We introduce the following method for solving (1.1): Consider the approximation $x_{n}$ of $x$ given by (2.3). Due to this method, we get the following 


$$
\sum_{i=1}^{n} \alpha_{i}\left\{-i U_{i-1}\left(t_{j}\right)+\frac{1}{n+1} \sum_{r=1}^{n}\left(1-t_{r}^{2}\right) h\left(t_{j}, t_{r}\right) U_{i-1}\left(t_{r}\right)\right\}=y\left(t_{j}\right), \quad 1 \leq j \leq n,
$$

where $t_{j}=\cos (j \pi / n+1)$.

THEOREM 3.1. If the following conditions

(i) $y \in C[-1,1], h \in C[-1,1 ;-1,1]$

(ii) equation (1.1) has a unique solution $x^{*} \in X$ for all $y \in X$

are satisfied, then, for $n$ sufficiently large, equation (3.1) has a unique solution $\alpha_{k}^{*}$,

$$
\left\|x^{*}-x_{n}^{*}\right\|_{\rho}=O\left\{E_{n}(y)_{C}+E_{n}^{t}(h)_{C}+E_{n}^{\tau}(h)_{C}\right\},
$$

where $E_{n}^{t}(f)_{C}=\inf \left\{\left\|f-P_{n}\right\|_{C}, P_{n}\right.$ is a polynomial of degree $\left.\leq n\right\}$.

Proof. Define the projection operator $P_{n}^{t}: C \rightarrow X$ by

$$
P_{n}^{t}(x)=\sum_{k=1}^{n} x\left(t_{k}\right) \frac{U_{n}(t)}{\left(t-t_{k}\right) U_{n}^{\prime}\left(t_{k}\right)} .
$$

Thus, equation (3.1) can be written in the equivalent form

$$
A_{n} x_{n}=G x_{n}+P_{n}^{t} H P_{n}^{\tau}\left(h x_{n}\right)=P_{n}^{t} y, \quad x_{n}, P_{n} y \in X_{n},
$$

where $X_{n}=\left\{x_{n}: x_{n}=\sum_{k=1}^{n} \alpha_{k} U_{k-1}(t), t \in[-1,1]\right\}$,

$$
\begin{aligned}
G x & =\frac{1}{\pi} \int_{-1}^{1} \frac{\sqrt{1-\tau^{2}} x(\tau)}{(\tau-t)^{2}} d \tau, \quad x \in X, \\
H h x & =\frac{1}{\pi} \int_{-1}^{1} \sqrt{1-\tau^{2}} h(t, \tau) x(\tau) d \tau .
\end{aligned}
$$

Since $P_{n}^{t} H P_{n}^{\tau}\left(h x_{n}\right)=P_{n}^{t} H\left(\left(P_{n}^{\tau} h\right) x_{n}\right)$, then, for all $x_{n} \in X_{n}$, we get

$$
\begin{aligned}
\left\|A x_{n}-A_{n} x_{n}\right\|_{\rho} & =\left\|H h x_{n}-P_{n}^{t} H P_{n}^{\tau}\left(h x_{n}\right)\right\|_{\rho} \\
& \leq\left\|\left(H h-P_{n}^{t} H h\right) x_{n}\right\|_{\rho}+\left\|P_{n}^{t} H\left(h-P_{n}^{\tau} h\right) x_{n}\right\|_{\rho} .
\end{aligned}
$$

According to [8], $\left\|P_{n}\right\| \leq C_{1},\left\|x-P_{n} x\right\|_{\rho} \leq C_{2} E_{n}(x)_{C}, x \in C[-1,1]$, we get

$$
\begin{aligned}
\left\|A x_{n}-A_{n} x_{n}\right\|_{\rho} & \leq C_{2} E_{n}\left(H h x_{n}\right)+C_{1}\left\|H\left(h-P_{n}^{\tau}\right) x_{n}\right\|_{C} \\
& =O\left\{E_{n}^{t}(h)_{C}+E_{n}^{\tau}(h)_{C}\right\}\left\|x_{n}\right\|_{\rho},
\end{aligned}
$$

so that

$$
\left\|A-A_{n}\right\|_{X_{n} \rightarrow X}=O\left\{E_{n}^{t}(h)_{C}+E_{n}^{\top}(h)_{C}\right\}=\epsilon_{n} .
$$

According to [1], for all $n$ such that $\left\|A^{-1}\right\| \epsilon_{n}<1, A_{n}$ has a bounded inverse and $\left\|A_{n}^{-1}\right\|=O(1), A_{n}: X_{n} \longrightarrow X_{n}$. Since $\left\|y-P_{n} y\right\|_{\rho}=O\left\{E_{n}(y)_{C}\right\}=\delta_{n}$. Finally, we have

$$
\begin{aligned}
\left\|x^{*}-x_{n}^{*}\right\|_{\rho} & =\left\|A^{-1} y-A_{n}^{-1} P_{n} y\right\|_{\rho} \\
& =O\left(\epsilon_{n}+\delta_{n}\right) \\
& =O\left\{E_{n}^{t}(h)_{C}+E_{n}^{\tau}(h)_{C}+E_{n}(y)_{C}\right\} .
\end{aligned}
$$


LEMmA 3.1. If $Q_{n}$ is an algebraic polynomial of degree $n-1$, then

$$
\left\|Q_{n}(t)\right\|_{C} \leq n \sqrt{\frac{n}{2}}\left\|Q_{n}\right\|_{\rho}, \quad n \geq 2
$$

Proof. One may write $Q_{n}$ as $Q_{n}(t)=\sum_{k=1}^{n} C_{k-1}\left(Q_{n}\right) U_{k-1}(t), n \in \mathbb{N}$, where $C_{j}\left(Q_{n}\right)$ $=\left(Q_{n}, U_{j}\right)_{\rho}$. Since $\left|U_{k-1}\right| \leq k$ it follows that

$$
\begin{aligned}
\left\|Q_{n}(t)\right\|_{C} & \leq\left\{\sum_{k=1}^{n} C_{k-1}\left|\left(Q_{n}\right)^{2}\right|\right\}^{1 / 2}\left\{\sum_{k=1}^{n} k^{2}\right\}^{1 / 2} \\
& =\left\|Q_{n}\right\|_{\rho} \sqrt{n(n+1)(2 n+1) / 6} \\
& \leq n \sqrt{n / 2}\left\|Q_{n}\right\|_{\rho} \\
& =\left\|Q_{n}\right\|_{\rho} O\left(n^{3 / 2}\right) .
\end{aligned}
$$

Define $W^{r} H_{\alpha}=\left\{x: x^{(r-1)}\right.$ is absolutely continuous, $\left.x^{(r)} \in H_{\alpha}\right\}$.

THEOREM 3.2. Assume that conditions (i) and (ii) of Theorem 3.1 are satisfied,

$$
y(t) \in W^{r} H_{\alpha}, h(t, \tau) \in W^{r} H_{\alpha}, \quad r \geq 0,0<\alpha \leq 1,
$$

then

$$
\begin{aligned}
& \left\|x^{*}-x_{n}^{*}\right\|_{\rho}=O\left(n^{-r-\alpha}\right), \\
& \left\|x^{*}-x_{n}^{*}\right\|_{C}=O\left(n^{3 / 2-r-\alpha}\right) .
\end{aligned}
$$

Proof. Since $y(t) \in W^{r} H_{\alpha}, h(t, \tau) \in W^{r} H_{\alpha}$, then, according to [7], one has $E_{n}(y)$ $=O\left(n^{-r-\alpha}\right), E_{n}^{t}(h)=O\left(n^{-r-\alpha}\right), E_{n}^{\tau}(h)=O\left(n^{-r-\alpha}\right), r+\alpha>0$. This proves (3.13). It is easy to show that

$$
\begin{aligned}
\left\|x^{*}-x_{n}^{*}\right\|_{C} & =\sum_{k=1}^{\infty}\left\|x_{2^{k} n}^{*}-x_{2^{k-1} n}^{*}\right\|_{C} \\
& \leq \sum_{k=1}^{\infty}\left(2^{k} n\right)^{(3 / 2)}\left[\left\|x^{*}-x_{2^{k} n}^{*}\right\|_{\rho}+\left\|x^{*}-x_{2^{k-1} n}^{*}\right\|_{\rho}\right] \\
& \leq C_{3} \sum_{k=1}^{\infty}\left(2^{k} n\right)^{(3 / 2)}\left(2^{k} n\right)^{-r-\alpha}=C_{4} n^{-r-\alpha+3 / 2},
\end{aligned}
$$

where $C_{3}, C_{4}$ are constants. This proves (3.14).

Define $C_{\rho}[-1,1]=\left\{x: \sqrt{1-t^{2}} x \in C[-1,1]\right\}$ and $\|x\|_{C_{\rho}}=\max \left\{\sqrt{1-t^{2}}|x(t)|\right\}$.

LEMMA 3.2. If $Q_{n}$ is a polynomial of degree $n-1$, then

$$
\left\|Q_{n}(t)\right\|_{C_{\rho}} \leq \sqrt{n}\left\|Q_{n}\right\|_{X} .
$$

Proof. Since $\left|U_{m}(t)\right| \leq\left(1-t^{2}\right)^{-1 / 2},-1 \leq t \leq 1, m=0,1, \ldots$, then

$$
\begin{aligned}
\sqrt{1-t^{2}}\left|Q_{n}(t)\right| & \leq \sum_{k=1}^{n}\left|C_{k-1}\left(Q_{n}\right)\right| \\
& \leq\left\{\sum_{k=1}^{n}\left|C_{k-1}\left(Q_{n}\right)\right|^{2}\right\}^{1 / 2} \sqrt{n}=\sqrt{n}\left\|Q_{n}\right\|_{\rho} .
\end{aligned}
$$


THEOREM 3.3. If $\left\|x^{*}-x_{n}^{*}\right\|_{X}=O\left(n^{-m}\right), m \in \mathbb{R}$, then

$$
\left\|x^{*}-x_{n}^{*}\right\|_{C_{\rho}}=O\left(n^{1 / 2-m}\right), \quad m>\frac{1}{2},
$$

Proof. Using Lemma 3.2 and the same technique as in Theorem 3.2, one obtains (3.18).

Conclusion. For the Mechanical Quadratures methods, the rate of convergence in space $C_{\rho}[-1,1]$ is better than that given in space $C[-1,1]$.

4. Approximation by degenerate kernels. We approximate $h(t, \tau)$ by

$$
h_{n}(t, \tau)=\sum_{k=1}^{n} a_{k}(t) b_{k}(\tau), \quad-1 \leq t, \tau \leq 1,
$$

where $\left\{a_{k}\right\},\left\{b_{k}\right\}$ are two sets of linearly independent functions. By substituting back into (1.1), we obtain

$$
A_{n} x=G x+\int_{-1}^{1} \sqrt{1-\tau^{2}} h_{n}(t, \tau) x(\tau) d \tau=y .
$$

(4.2) can be written as

$$
G x+\sum_{k=1}^{n} \alpha_{k} a_{k}=y
$$

where $\alpha_{k}=\int_{-1}^{1} \sqrt{1-\tau^{2}} x(\tau) b_{k}(\tau) d \tau$. Then the solution of (4.3) is given by

$$
\begin{gathered}
x=G^{-1} y-\sum_{k=1}^{n} \alpha_{k} G^{-1} a_{k}, \\
G^{-1} y=-\sum_{k=1}^{\infty} \frac{\left(y, U_{k-1}\right)_{\rho}}{k} U_{k-1}(t) .
\end{gathered}
$$

Multiplying (4.4) by $\sqrt{1-t^{2}} b_{j}(t)$ and integrating, we get the linear system of equations

$$
\alpha_{j}+\sum_{k=1}^{n} \gamma_{j k} \alpha_{k}=y_{j}, \quad 1 \leq j \leq n,
$$

where $\gamma_{j k}=\int_{-1}^{1} \sqrt{1-t^{2}} b_{j} G^{-1}\left(a_{k}\right) d t, y_{j}=\int_{-1}^{1} \sqrt{1-t^{2}} b_{j} G^{-1}(y) d t$. Define $q=\sqrt{1-t^{2}}$ $\sqrt{1-\tau^{2}}$.

THEOREM 4.1. Suppose that

(i) $y \in L_{2, \rho}$

(ii) $h \in L_{2, q}[-1,1 ;-1,1]$

(iii) $\epsilon_{n}^{2}=\int_{-1}^{1} \int_{-1}^{1} q(t, \tau)\left|h(t, \tau)-h_{n}(t, \tau)\right|^{2} d t d \tau \longrightarrow 0, n \rightarrow \infty$

(iv) equation (1.1) has a unique solution.

Then for all $n$ such that $q_{n}=\epsilon_{n}\left\|A^{-1}\right\|<1, A: X \rightarrow X$, the linear system of equations (4.5) has a unique solution $\left\{\alpha_{k}^{*}\right\}_{1}^{n}$ and the approximate solution $x_{n}^{*}=G^{-1}(y)-$ $\sum_{k=1}^{n} \alpha_{k}^{*} G^{-1}\left(a_{k}\right)$ converges to the exact solution $x^{*},\left\|x^{*}-x_{n}^{*}\right\|_{\rho}=O\left(\epsilon_{n}\right)$. 
Proof. For $x \in X$, we have

$$
\begin{aligned}
\left\|A x-A_{n} x\right\|_{\rho} & =\left\|\int_{-1}^{1} \sqrt{1-\tau^{2}}\left[h(t, \tau)-h_{n}(t, \tau)\right] x(\tau) d \tau\right\|_{\rho} \\
& \leq\|x\|_{\rho}\left\|h-h_{n}\right\|_{L_{2, q}}=\epsilon_{n}\|x\|_{\rho} .
\end{aligned}
$$

Then $\left\|A-A_{n}\right\|_{X \rightarrow X} \leq \epsilon_{n}$, according to [6] (4.5) has a unique solution $\left\{\alpha_{k}^{*}\right\}_{1}^{n}$, $\left\|x^{*}-x_{n}^{*}\right\|=O\left(\epsilon_{n}\right)$.

\section{REFERENCES}

[1] B. G. Gabdulkhaev, Optimalnye approksimatsii reshenii lineinykh zadach. [Optimal approximations of solutions of linear problems], Kazan. Gos. Univ., Kazan, 1980 (Russian). MR 83k:65047.

[2] M. A. Golberg, The convergence of several algorithms for solving integral equations with finite-part integrals, J. Integral Equations 5 (1983), no. 4, 329-340. MR 85h:65273. Zbl 529.65079.

[3] _ The convergence of several algorithms for solving integral equations with finite part integrals. II, J. Integral Equations 9 (1985), no. 3, 267-275. MR 87j:65162a. Zbl 606.65090.

[4] J. Hadamard, Lectures on Cauchy's problem in linear partial differential equations, Dover Publications, New York, 1952. MR 14,474f. Zbl 049.34805.

[5] N. I. Ioakimidis, Two methods for the numerical solution of Bueckner's singular integral equation for plane elasticity crack problems, Comput. Methods Appl. Mech. Engrg. 31 (1982), no. 2, 169-177. MR 83i:73052. Zbl 484.73074.

[6] L. V. Kantorovic and G. P. Akilov, Funktsionalnyi analiz v normirovannykh prostranstvakh. [Functional analysis in normed spaces], Gosudarstv. Izdat. Fis. Mat. Lit., Moscow, 1959 (Russian). MR 22\#9837. Zbl 127.06102.

[7] G. Meinardus, Approximation of functions: Theory and numerical methods., Expanded translation of the German edition. Translated by Larry L. Schumaker. Springer Tracts in Natural Philosophy, vol. 13, Springer-Verlag, New York, 1967. MR 36\#571. Zbl 152.15202.

[8] A. F. Timan, Teorij pribli+enij funkcii deistvitel'nogo peremennogo. [Theory of approximation of functions of a real variable], Gosudarstv. Izdat. Fiz. Mat. Lit., Moscow, 1960 (Russian). MR 22\#8257. Zbl 117.29001.

[9] D. E. Williams, Some mathematical methods in three-dimentional subsonic flutterderivatives theory, British Ministry of Aviation Reports and Memo, No.3302 (1961).

Ashour: Department of MAThematics, CAiro University, Giza, EgyPT 


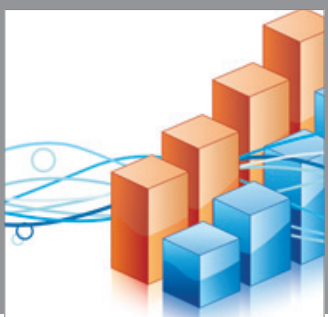

Advances in

Operations Research

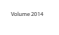

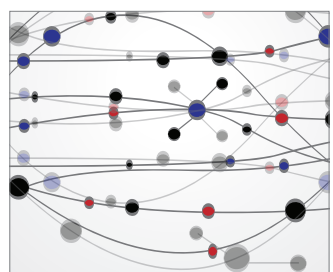

\section{The Scientific} World Journal
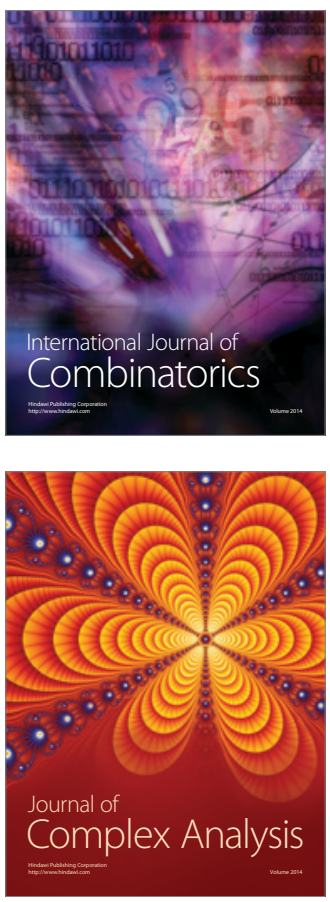

International Journal of

Mathematics and

Mathematical

Sciences
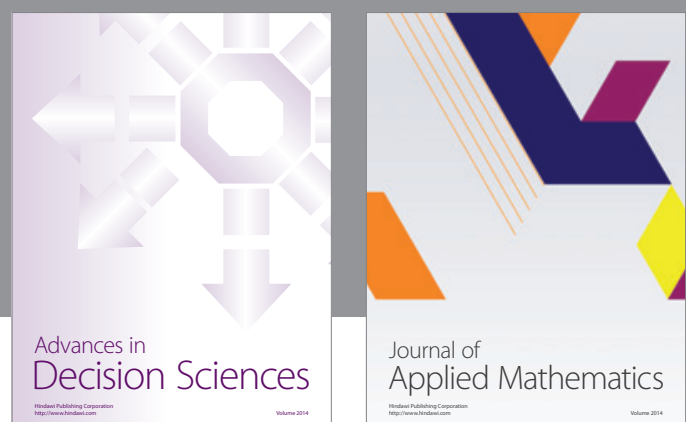

Journal of

Applied Mathematics
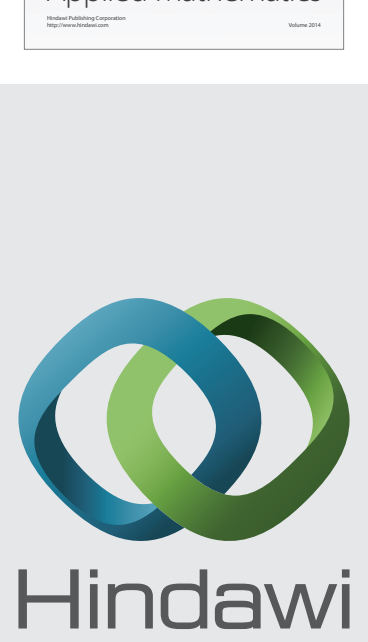

Submit your manuscripts at http://www.hindawi.com
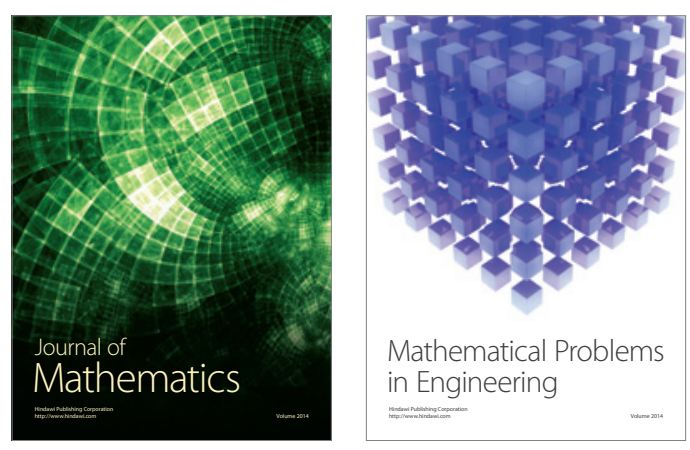

Mathematical Problems in Engineering
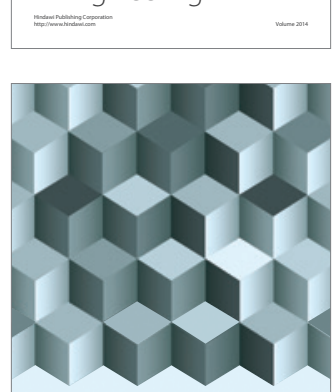

Journal of

Function Spaces
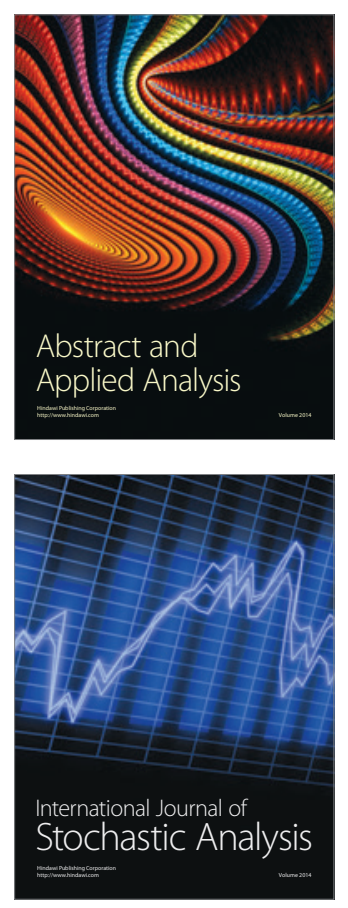

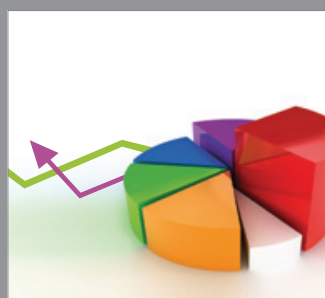

ournal of

Probability and Statistics

Promensencen
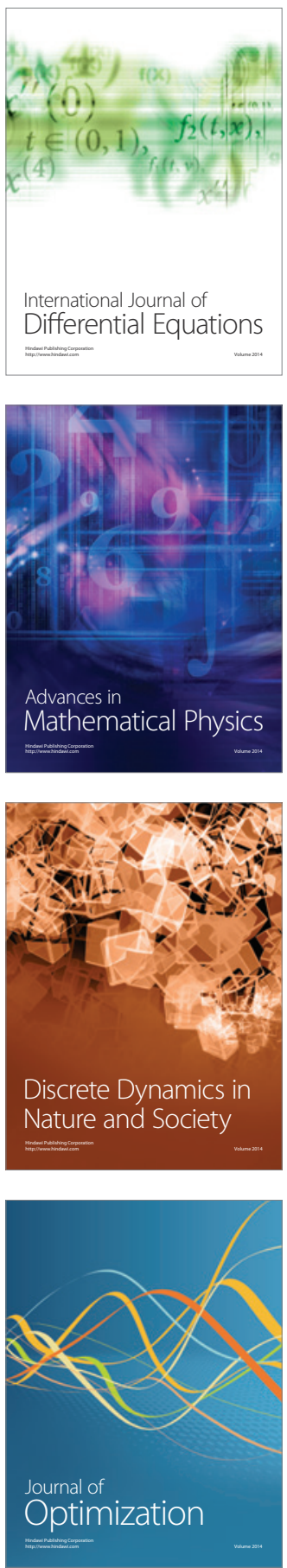